\title{
Recurrent major umbilical bleeding caused by omental varices in two patients with umbilical hernia and portal hypertension
}

\author{
Ioannis Sarantitis, ${ }^{1}$ Hywel Satherley, ${ }^{1}$ Haren Varia, ${ }^{2}$ Stephen Pettit ${ }^{1}$
}

${ }^{1}$ Department of Surgery, Blackpool Victoria Hospital, Blackpool, UK

${ }^{2}$ Department of Radiology, Blackpool Victoria Hospital, Blackpool, UK

\section{Correspondence to} Stephen Pettit, stephenhpettit@btinternet.com

Accepted 20 July 2015

\footnotetext{
To cite: Sarantitis I, Satherley $\mathrm{H}$, Varia $\mathrm{H}$, et al. BMJ Case Rep Published online: [please include Day Month Year] doi:10.1136/ bcr-2015-209647
}

\section{SUMMARY}

We describe two cases of recurrent major umbilical bleeding in male patients with Childs A liver cirrhosis and portal hypertension. Both patients had umbilical hernias with omentum incarcerated in the hernial sac. In both cases, portosystemic abdominopelvic CT scan had shown omental varices without significant paraumbilical cutaneous varices. Previous reports have shown that venous communication can develop between omental varices and overlying abdominal wall scars, resulting in spontaneous cutaneous bleeding. We concluded that there was a similar mechanism for the umbilical bleeding in our cases and both were successfully treated by excising the umbilicus, ligating the omental varices within the hernia sac, returning the omentum to the abdominal cavity and repairing the hernia defect. This cause for umbilical bleeding has not been previously reported. We advise that with similar cases, excision of the umbilicus and ligation of the omental varices is safe and effective treatment.

\section{BACKGROUND}

Umbilical bleeding is a well recognised but rare complication of portal hypertension. In previously reported cases, it has arisen from rupture of umbilical varices that have developed due to portosystemic communication between the recanalised umbilical vein and the periumbilical veins of the abdominal wall. ${ }^{1-8}$ We present two cases of recurrent major umbilical bleeding from omental varices incarcerated within an umbilical hernia. Both cases were treated successfully with excision of the umbilicus and ligation of the omental varices. This is the first report of umbilical bleeding from omental varices in patients with umbilical hernias and portal hypertension. We wished to raise awareness of this potentially life-threatening condition and have discussed its pathogenesis, investigation and surgical management.

\section{CASE PRESENTATION}

\section{Case 1}

A 51-year-old man presented with spontaneous bleeding from his umbilicus. He described blood pouring out of his umbilicus as if a tap had been turned on (figure 1). His clothes had become soaked with blood. He had Childs A alcohol-induced liver cirrhosis with portal hypertension and a body mass index of 53. He was admitted to hospital and the bleeding was controlled with direct pressure over his umbilicus. He was discharged home 3 days later with a haemoglobin level of $112 \mathrm{~g} / \mathrm{L}$, as there had been no further bleeding. However, he was readmitted later the same day with a further major umbilical bleed and his haemoglobin had fallen to $72 \mathrm{~g} / \mathrm{L}$. He was transfused and a portovenous abdominopelvic CT scan was performed to define the portal venous anatomy and source of umbilical bleeding. It showed that bleeding had arisen from omental varices within the umbilical hernia. Three-dimensional (3D) images were reformatted for surgical planning. Surgery was performed the following day to prevent further bleeding. This involved excising his umbilicus, ligating the omental varices contained within the umbilical hernia and returning the omentum to the abdominal cavity. The hernia was then repaired. The patient made a good recovery and has been followed up for over 6 months; he has had no further bleeding.

\section{Case 2}

A 48-year-old man with Childs A alcohol-induced liver cirrhosis was referred to the surgical clinic by his gastroenterologist following recurrent episodes of major umbilical bleeding over the preceding 10 months. This was often precipitated by mild exertion, such as walking up stairs, and the bleeding was usually only noticed when blood had run down his legs to pool on the floor (figure 2). He could control the bleeding by applying pressure over his umbilicus. An MRI scan had been performed to assess the severity of his cirrhosis. On examination, he had a non-reducible umbilical hernia. There were no paraumbilical cutaneous varices and there was no audible venous hum around his umbilicus. A

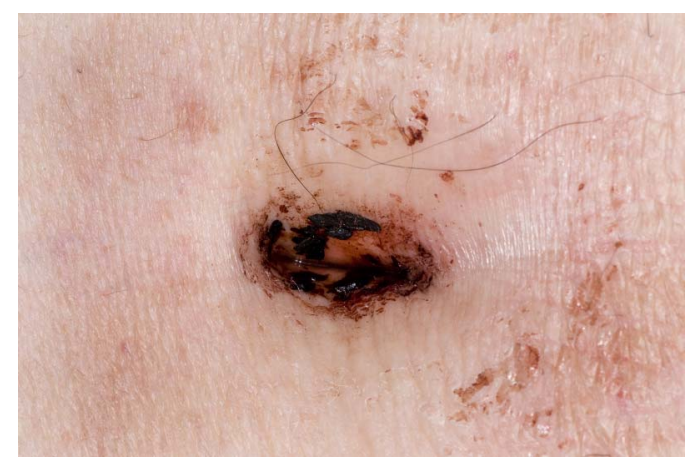

Figure 1 Operative photograph of umbilicus in case 1 showing old blood in and around the umbilicus. 


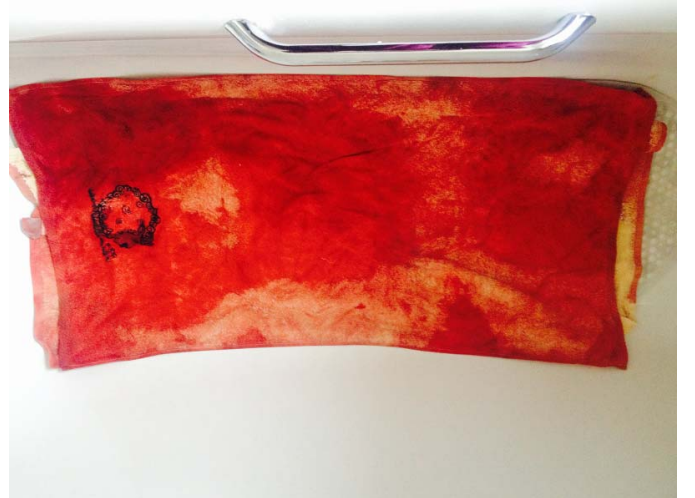

Figure 2 Photograph showing one of several blood stained towels in a bath that had been used to remove blood from the floor after one umbilical bleed in case 2 .

portovenous abdominopelvic CT scan was performed to define the portal venous anatomy and source of umbilical bleeding. It showed that bleeding had arisen from omental varices within the umbilical hernia. The 3D images were reformatted for surgical planning and elective surgery was performed the following week to prevent further bleeding. This involved excising the patient's umbilicus, ligating the omental varices contained within the hernia and returning the omentum to the abdominal cavity. The hernia was then repaired. The patient made a good recovery and has been followed up for over 5 months; he has had no further bleeding.

\section{INVESTIGATIONS}

Haematological and biochemical investigation in Case 1 was consistent with established cirrhosis: albumin $22 \mathrm{~g} / \mathrm{L}$ (35-50), bilirubin $25 \mu \mathrm{mol} / \mathrm{L}(3-20)$, platelets $80 \times 10^{9} / \mathrm{L}(150-400)$ and international normalised ratio (INR) 1.3 (1.0-1.1). A CT scan showed cirrhosis with portal hypertension and recanalisation of the umbilical vein. There was no ascites and no evidence of significant paraumbilical cutaneous varices. An umbilical hernia was noted containing omentum and omental varices were present (figure 3).

Investigations in Case 2 were also consistent with established cirrhosis: albumin $35 \mathrm{~g} / \mathrm{L}$, bilirubin $41 \mu \mathrm{mol} / \mathrm{L}$, platelets $45 \times 10^{9} / \mathrm{L}$ and INR 1.3. An MRI scan showed cirrhosis and portal hypertension with a trace of ascites overlying the patient's liver. There was recanalisation of his umbilical vein but no significant cutaneous paraumbilical veins. Omental varices were present (figure 4). A portovenous abdominopelvic CT scan was also performed, which showed that his umbilical hernia contained omentum and omental varices were present within the hernia.

\section{DIFFERENTIAL DIAGNOSIS}

In previously reported cases of spontaneous umbilical bleeding in patients with cirrhosis and portal hypertension, the bleeding has originated from rupture of umbilical varices caused by portosystemic communication between the recanalised umbilical vein and periumbilical veins of the abdominal wall. ${ }^{1-8}$ We dismissed this possibility in our two cases as, when examined, they did not have caput medusae or a periumbilical venous hum, and CT and MRI showed no evidence of significant periumbilical cutaneous varices. The significant abnormality in both cases was the presence of an umbilical hernia containing incarcerated omentum and the presence of omental varices within the

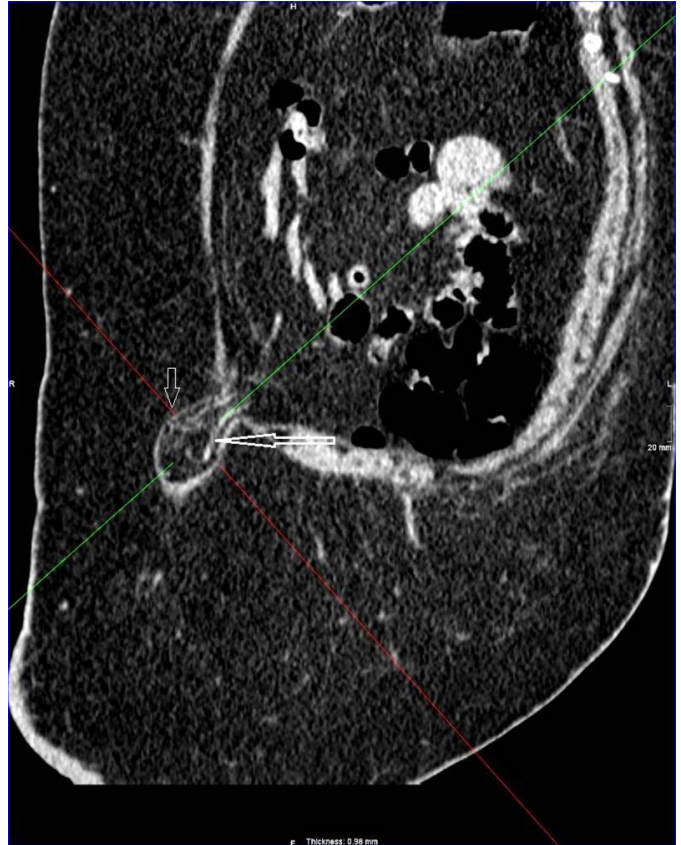

Figure 3 Coronal oblique CT image in case 1 showing the umbilical hernia (short arrow) containing omental varices (long arrow).

umbilical hernia. Previous reports have described spontaneous bleeding from abdominal wall scars in patients with portal hypertension where the bleeding has arisen from venous communications between the scar and underlying adherent omental varices. ${ }^{9}$ This led us to believe that in both cases the umbilical bleeding originated from omental varices incarcerated within the umbilical hernia.

\section{TREATMENT}

Both cases were treated with excision of the umbilicus, ligation of the omental varices contained within the hernia and return of the omentum to the abdominal cavity (figures 5 and 6). The umbilical defect was then repaired and the skin closed with subcuticular sutures. In both cases, omental varices had become adherent to the base of the umbilicus allowing venous communications to develop between the varices and cutaneous veins at the base of the umbilicus.

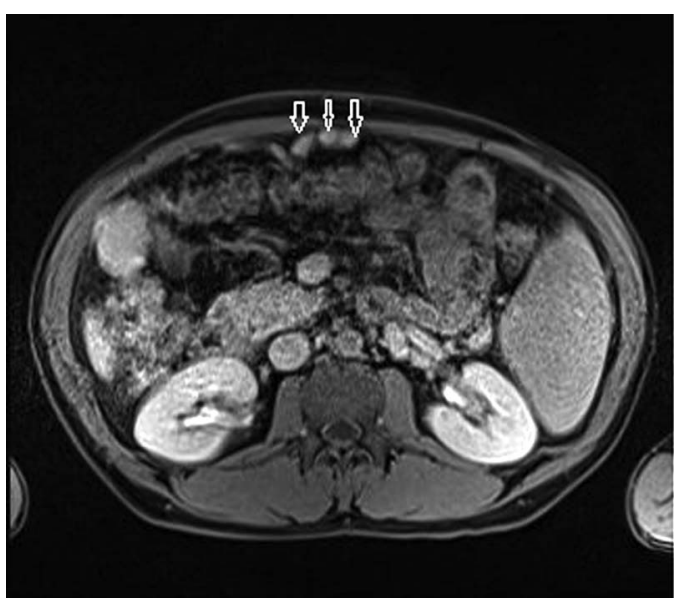

Figure 4 MRI scan in case 2 showing omental varices (arrows). 


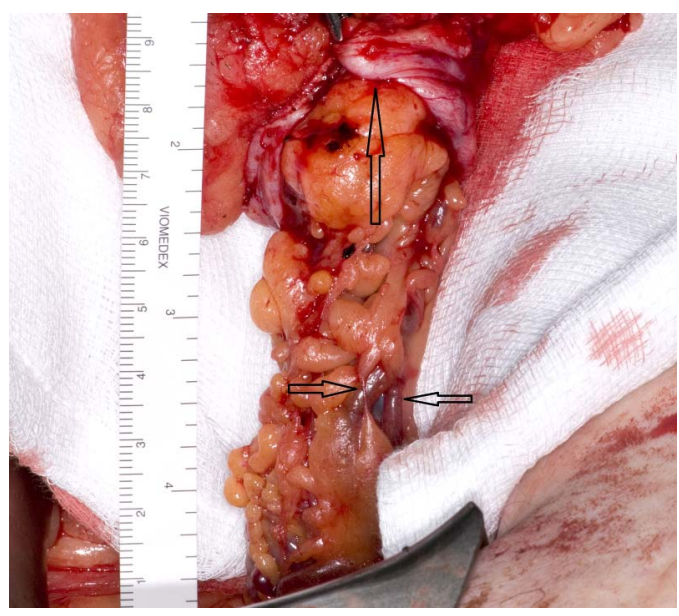

Figure 5 Operative photograph in case 1. Short arrow shows omental varices, long arrow shows base of the umbilicus.

\section{OUTCOME AND FOLLOW-UP}

Both patients made an uneventful recovery. Case 1 was discharged to convalescence 4 weeks later. He required an extended hospital stay because of mobility issues caused by his obesity. He returned home 7 weeks after surgery. He has been followed up for over 6 months and has had no further bleeding. Case 2 was discharged on the day of surgery. He has been followed up for over 5 months and has had no further bleeding.

\section{DISCUSSION}

In patients with cirrhosis of the liver, portal venous hypertension develops due to progressive fibrosis and regenerating nodules obstructing the portal circulation within the liver sinusoids. This results in the development of portosystemic venous connections, which decompress the portal venous system. The collateral circulation develops as a result of vasodilation, vascular remodelling and angiogenesis. This produces varices, high pressure veins that are most commonly seen in the oesophagus, though other splanchnic veins can become varicose including those of the omentum. Bleeding from oesophageal varices is a common gastroenterological problem in cirrhotic patients, and treatment is usually with the use of vasoactive drugs to reduce the splanchnic blood flow, such as terlipressin and octreotide, followed by injection sclerotherapy or banding of the varices. In $20 \%$ of patients with cirrhosis and portal hypertension, the left portal vein communicates with the recanalised umbilical vein in the falciform ligament. This then causes high pressure dilated

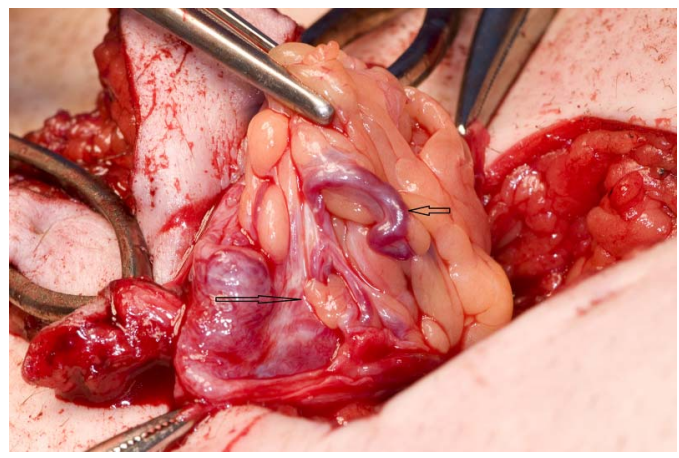

Figure 6 Operative photograph in case 2. Short arrow shows omental varices, long arrow shows base of the umbilicus. veins around the umbilicus (umbilical varices), which communicate with the superficial and deep epigastric veins draining into the external iliac veins. This situation is known as Cruveilhier-Baumgarten syndrome. ${ }^{10}$ Approximately 5\% of these patients develop a network of dilated veins around the umbilicus giving a caput medusae appearance and audible venous hum. ${ }^{11}$ Spontaneous umbilical bleeding has been reported from umbilical varices ${ }^{1-4}$ and has been treated with transjugular intrahepatic portosystemic shunt, ${ }^{5}$ sclerotherapy, ${ }^{6}$ laparotomy and ligation of the recanalised umbilical vein, ${ }^{7}$ and laparoscopically assisted ligation of the umbilical vein and epigastric veins ${ }^{8}$ to decompress the periumbilical varices. Spontaneous cutaneous bleeding from abdominal scars has also been reported in patients with cirrhosis and portal hypertension. ${ }^{9}$ This occurs when venous communications develop between veins in the cutaneous scar and underlying adherent omental varices. ${ }^{9}$ These cases have been treated with mesocaval interposition shunt and sclerotherapy. ${ }^{9}$

Our two cases of spontaneous recurrent major umbilical bleeding did not have caput medusae or a venous periumbilical hum, and imaging (CT and MRI) did not show significant periumbilical variceal veins despite the umbilical veins having recanalised. The common radiological abnormality in both patients was the presence of an umbilical hernia containing incarcerated omentum with omental varices. Radiological advice was taken to identify the most appropriate imaging modality to identify the source of umbilical bleeding. In both cases, portovenous abdominopelvic CT scanning was advised and performed, as it is an easily accessible and quick investigation with good resolution of venous anatomy. MRI was not used as it takes much longer to perform and patient movement during scanning may degrade the quality of the venous images obtained. Angiography was not considered useful in our cases as arterial bleeding from the umbilicus was not suspected and arterial embolisation was not contemplated. The portovenous abdominopelvic CT imaging led us to believe that the umbilical bleeding originated from the omental varices contained within the umbilical hernias due to the development of venous communications between the omental varices incarcerated in the umbilical hernia and cutaneous veins in the base of the umbilicus. Case 2 had noticed that the umbilical bleeding often occurred after he had walked up stairs. It seems likely that contraction of his abdominal muscles during this exercise constricted the margins of his umbilical hernia, compressing the incarcerated omentum within the hernia. This would result in a rise in venous pressure in the trapped omental varices, which would open collateral veins into the base of the umbilicus. Once started, the venous bleeding would then continue until pressure was applied to the umbilicus. Other causes of a temporary rise in intra-abdominal pressure, such as coughing or straining, could also precipitate episodes of umbilical bleeding. As a result of our clinical and radiological evaluation, we opted to treat both patients with excision of the umbilicus and ligation of the omental varices contained within the umbilical hernia. Portovenous abdominopelvic CT scanning was undertaken prior to surgery to define the portal venous anatomy and source of bleeding, and 3D images were reformatted for surgical planning. The operative findings in both cases were the same. When the umbilical hernia sacs were opened, dilated omental variceal veins were found densely adherent to the base of the umbilicus (figures 5 and 6). The omentum was separated from the base of the umbilicus, the omental varices were ligated and the omentum was returned to the abdominal cavity. The hernia was then repaired. There has been no further bleeding in either case with over 5 months 


\section{Patient's perspective}

\section{Case 1:}

I had my first bleed on 5 November 2014 at roughly 23:00. I was walking to the bathroom and suddenly I saw blood on the floor. I was trying to figure out where the bleeding was coming from and I then realised it was my naval. I was confused but also very scared, as I was not in any pain whatsoever. I tried to stop the bleeding by holding a towel over my naval applying pressure. The bleeding failed to stop. Consequently, I telephoned for an ambulance and was taken to A\&E.

The bleeding failed to stop for roughly $45 \mathrm{~min}-60 \mathrm{~min}$. I was kept in overnight to be monitored, as I was advised the nurses and doctors were unaware of what the problem was. My next bleed was at 04:30 on the morning of 6 November 2014. This started out of nowhere as I was in bed at the time. The bleed lasted for approximately 35-40 min and my naval was then packed to prevent further bleeding. Later that day, I was taken down for an ultrasound scan and the results failed to show any problems.

On 7 November 2014, I was seen by a consultant who discharged me to my home address with no explanation as to the bleeding. At 23:00 that evening, I was on my way to the bathroom and the bleeding began again. I applied pressure using towels, as done previously, and rang for an ambulance. I do not feel I should have been discharged from the hospital without any diagnosis. I was confused and upset about the bleeding, mainly due to the fact that I was unaware of why it was happening and because of the amount of blood I was losing. I felt like I was dying.

On arrival at the hospital that evening, they packed my naval again and managed to stop the bleeding. I was put on the same ward that the consultant who discharged me previously, worked on. I was quite upset that I was not approached by him with an apology or an explanation as to why I was sent home with no diagnosis, when he was fully aware there was a high chance of bleeding occurring again.

On 8 November 2014 at roughly 16:30, I had another bleed. When I refer to having a bleed, the blood actually gushes out my naval and cannot be stopped for a considerable amount of time. The nurses again packed my naval and managed to stop the bleeding. On that evening, I was given a blood transfusion ( $2 \mathrm{~L})$.

On 9 November 2014, I was taken down for a CT scan and was told I had an umbilical Hernia. I was happy to receive a diagnosis, however, I do feel I should have been sent down for this scan at an earlier stage, especially considering the amount of blood I had lost and the fact that this excessive bleeding had not been seen before.

On 10 November 2014, I was seen by SP. I was given the option of surgery, which involved a very high risk of death. However, there was no alternative as I was advised that the bleeding would not stop if I did not undergo surgery. I was extremely worried and scared about both options, however. I chose to undergo surgery to remove the umbilical hernia despite the risks involved. I was warned of a lower survival rate due to suffering from cirrhosis of the liver. I underwent surgery on 11 November 2014. The surgery was successful and I was well looked after and cared for on the ICU ward of the hospital.

I was then kept in hospital until 1 December 2014 as my recovery was being monitored. On 1 December 2014, I was admitted to Bispham Rehabilitation. I underwent physiotherapy as due to me being bed bound for such a long period of time I could not walk properly. I was discharged on 7 January 2015 and my wound is still healing.

\section{Case 2:}

The bleeding first occurred on 17/11/2013, I was due to leave for work after coming down the stairs and had sat in a chair to tie my laces. There was no pain and I only realised I was bleeding from my naval after it had seeped through all my clothing and was dripping on the floor. In my opinion, I lost approximately $1 / 2$ pt of blood.

An ambulance was called and I was admitted to $A \& E, B V H$ and then later admitted to AMU.

It was advised that they would be testing for HIV or a bone marrow problem. I was given a chest X-ray and advised I would have an ultrasound scan. I was seen also by an alcohol liaison nurse.

I was discharged on 19/11/13 without a scan but advised I would get an outpatient appointment.

On $21 / 11 / 13$, I was admitted to A\&E with further bleeding of approximately $1 / 3$ pt after walking down the stairs.

I was assessed by a doctor in A\&E and further blood was taken; I was advised that my platelet count was extremely low. No advice or explanations were given other than if it bled again, not to call an ambulance but to seek advice of a GP or attend a walk in centre. I felt extremely concerned as no scans had been made, and my stomach was extremely swollen and my navel was distended.

On 03/12/13, a voicemail was left on my phone advising me of an appointment at Gastro on 03/12/13. On attending, it turned out the appointment was for a follow-up with the alcohol liaison nurse. On discussion, it turned out that no follow-up appointments had been made for further consultation or for scans. I was advised to contact the medical secretary to discuss this further.

After contacting the hospital again and voicing concerns about the lack of treatment or advice I had been given, I was advised to attend AMU again on 5/12/13.

I was admitted to AMU on 5/12/13. I had slight bleed again prior to admission.

Further blood was taken and the doctor seemed surprised that no full examination or swabs of my naval had been made. I was also seen by two surgeons who stated that an operation was not an option as no scans had been completed and there was also infection. I was advised that I would stay overnight and have a scan.

I was discharged on 6/12/13 with a prescription for antibiotic cream; I had still not undergone a scan.

In between admissions, I had been to my GP who advised me that, in his opinion, I would need to see a surgeon, but his knowledge was limited. He was fully supportive though and gave me advice on compression to the bleeding area. He told me not to hesitate in calling an ambulance if bleeding did not stop, etc and also signed me off work. 
On 16/12/13, I received an appointment on 10/2/14. I had still not received further correspondence from BVH and had no scans. I contacted the receptionist at ultrasound scans who informed me that all requests had been cancelled on discharge. My GP finally arranged a scan for $2 / 1 / 14$ at a walk in centre.

On 10/2/14, I had my appointment with the consultant; the scans had been completed at the walk in centre and my GP confirmed that he could see the images. The consultant, however, stated that he could not see any scans but did not need them to diagnose stage A cirrhosis and that as long as no further alcohol was consumed and I ate a healthy diet there was a good possibility that I would be able to lead a normal healthy life. There had been spot bleeding since my admission prior to this appointment. My next appointment was for September.

Between February 2014 and the next appointment with the consultant there were numerous spot bleeds and episodes of seeping. Again there was no warning, and I was taking most of the time off work. I had numerous bleeds at work, with one major bleed, again after coming down the stairs. Each time, the bleeding eventually stopped by compression. I was extremely scared of being in public places in case of a bleed and my daughter's wedding was stressful because of this condition. I was diagnosed by my GP as having depression but could not be medicated due to the effect the medication would have on my blood. I also became reclusive.

I had also been referred at this time, by the consultant, for gastroscopy, ultrasound and MRI scan.

At my 9/9/14 appointment, the consultant advised me that, he was not an expert, he could see no or very few signs of a hernia. My GP, at my request, had written to the consultant enquiring what was to be done about my bleeding, etc. The consultant replied to my GP on 3/09/14 stating that treatment options were limited. He stated that there was certainly no role for medical treatment in my case but that if I continued to get consistent bleeding it might be worthwhile obtaining a surgical opinion. He arranged to get me back to clinic to have a look and decide the most appropriate course of action and, of course, refer me to a surgeon if it was felt to be the next appropriate strategy.

I was advised that the consultant would discuss further with a surgeon.

On 11/09/14, after sneezing, there was a serious bleed. Compression could not stop the bleeding and so an ambulance was called for. I was admitted to A\&E and advised by the doctor there that something should be done about my condition (she was extremely sympathetic), I was kept on observation ward overnight—I felt that I was treated extremely badly—not cleaned up — covered in blood, without blankets or pillow, with a drip kept in my arm for excessive lengths of time.

Before discharge I was spoken to by a triage surgeon who stated that, in his opinion, surgery could carry more risks and that the bleeding was something that I would probably learn to live with. I explained that we were awaiting an appointment with a surgeon and he advised me that he would speak to the consultant. Advice was given to stem bleeding by putting gauze on my finger and pushing in the naval.

On 23/9/14, 24/9/14 and again on 25/9/14, I bled but stopped it by applying pressure; bleeding seemed to occur after movement, for instance, while getting out of bed.

On 25/9/14, I called the secretary as I was feeling really confused and still had not received notification from BVH for any further appointments. The secretary was really helpful and explained that she had not received any notification of my case. There was no correspondence on the system apart from the consultant's letter of 3/09/14.

A provisional appointment was made to see a surgeon on 17/12/14.

There was bleeding again on 4/10/14 and 23/10/14.

On 8/11/14 I called 111 as I was feeling extremely ill with lower back pain; I was admitted to AMU—but this turned out to be a water infection. (The on call GP was extremely helpful and concerned about the lack of intervention in my case).

There was spot bleeding occurring sporadically_oozing up to the date of seeing yourself.

I have found the whole experience extremely distressing with no advice, no monitoring and no blood taken (except on admittance to hospital or by my GP). Initially, it felt as if my case was judged as just another alcohol-related condition. In my opinion, until my appointment with yourself, there was no communication within the hospital or any procedures carried out while on the wards. I have been off work for most of the year and am at risk of losing my job. I feel that the amount of blood that I was losing was completely dismissed, once even being told that a small amount goes a long way. If it was not for the constant phone calls made by my wife, I do not even think that it would have got to the point of seeing yourself.

It got to the point that I thought I was going to die from bleeding, I did not leave the house, drive, or even come down the stairs. I still have concerns that my blood is not being monitored and my next appointment with the consultant is not until May.

On the last appointment, after being asked if I had bled since the operation, I was advised that there was nothing to say that I could not bleed from elsewhere.

Since the operation, I am attempting to regain my confidence and build up my mobility as the mindset is that it can still bleed. I am eternally grateful for your support and the operation you performed on me. It was not until my meeting with you that anything had been explained. I wish looking back that this could have happened much earlier. There was so much more I could write but a lot is now hazy as so much has happened.

follow-up in both cases. Case 1 has lost some weight through diet and exercise, however, there is a high likelihood of incisional hernia development due to failure of the umbilical hernia repair because of his morbid obesity. This may expose him to similar risk of further bleeding. In this situation, as he has Childs A cirrhosis, a transjugular intrahepatic portosystemic shunt would be considered to reduce the risk of rebleeding.
There may also be a role for $\beta$ blockers in this situation, as they have been shown to reduce the risk of variceal bleeding in some cases by reducing cardiac output and producing splanchnic vasoconstriction, thereby reducing portal flow and portal pressure.

Spontaneous umbilical bleeding is a well recognised but rare complication of portal hypertension, and this can be lifethreatening. ${ }^{2}{ }^{4}$ This is the first report of spontaneous umbilical 


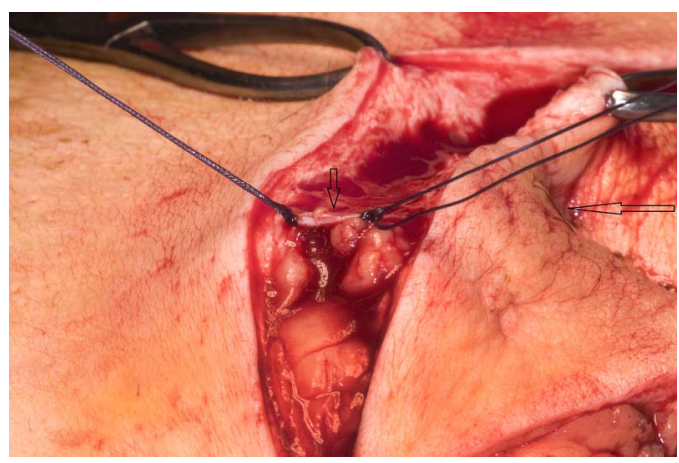

Figure 7 Operative photograph showing a periumbilical vein being ligated (short arrow) during excision of the umbilicus (long arrow) in case 1.

bleeding arising in patients with cirrhosis and portal hypertension where the bleeding has originated from omental varices being incarcerated in an umbilical hernia. Surgeons are reluctant

\section{Learning points}

- Umbilical bleeding in patients with cirrhosis and portal hypertension may be from periumbilical cutaneous varices.

- Umbilical bleeding in patients with cirrhosis and portal hypertension who have umbilical hernias containing omentum may be from omental varices that have established a venous communication with cutaneous veins in the base of the umbilicus.

- Emergency treatment of umbilical bleeding in patients with cirrhosis and portal hypertension is to control the bleeding with pressure over the umbilicus, to transfuse as necessary and to consider the use of vasoactive drugs such as terlipressin and octreotide to reduce the splanchnic blood flow.

- A portovenous abdominopelvic CT scan should be performed in patients with cirrhosis and portal hypertension who have recurrent umbilical bleeding, to identify the portal venous anatomy and site of bleeding.

- Excision of the umbilicus and ligation of omental varices is a safe and effective treatment for patients presenting with umbilical bleeding arising from omental varices incarcerated in an umbilical hernia. to perform umbilical hernia repair in patients with portal hypertension because of the perceived danger of major venous bleeding from periumbilical varices. In both our cases, preoperative imaging did not demonstrate significant periumbilical varices, and surgery was uneventful and without unexpected venous bleeding from the periumbilical incision (figure 7).

We advise that portosystemic abdominopelvic CT scanning should be performed in patients presenting with recurrent major umbilical bleeding who have cirrhosis and portal hypertension together with irreducible umbilical hernias, to identify the portal venous anatomy. This will demonstrate whether omental varices are present within the umbilical hernia and whether significant periumbilical varices are present. If there are no significant periumbilical varices and omental varices are confirmed within the umbilical hernia, then it can be assumed that the bleeding originates from omental varices within the umbilical hernia. In this situation, excision of the umbilicus with ligation of the omental varices is a safe and effective treatment.

Contributors IS and HS identified this case as worthy of publication because of its clinical importance. SP was in charge of the case and HV undertook the radiological investigations.

Competing interests None declared.

Patient consent Obtained.

Provenance and peer review Not commissioned; externally peer reviewed.

\section{REFERENCES}

1 Douglas JG. Umbilical haemorrhage--an unusual complication of cirrhosis. Postgrad Med J 1981;57:461-2

2 Bahner DR Jr., Holland RW III. Exsanguinating hemorrhage from a caput medusae: cutaneous variceal bleeding. J Emerg Med 1992;10:19-23.

3 Lewis CP, Murthy S, Webber SM, et al. Hemorrhage from recanalized umbilical vein in a patient with cirrhosis. Am J Gastroenterol 1999;94:280.

4 Hoi KY, Mignanelli ED, Lightfoot D. Fatal haemorrhage from a caput medusae: a differential to a stabbing. Emerg Med Australas 2007;19:173-5.

5 Fitzgerald JB, Chalmers N, Abbott $G$, et al. The use of TIPS to control bleeding caput medusae. Br J Radiol 1998;71:558-60.

6 Assis DN, Pollak J, Schilsky ML, et al. Successful treatment of a bleeding umbilical varix by percutaneous umbilical vein embolization with sclerotherapy. J Clin Gastroenterol 2012;46:115-18.

7 Hashash JG, Borhani A, Rabinovitz M, et al. Umbilical Varix in a Cirrhotic Patient. ACG Case Rep J 2014;2:1.

8 Zachariah SK, Krishnankutty SL, Raja N. Laparoscopic management of massive spontaneous external haemorrhage from the umbilical varix due to recanalisation of the paraumbilical vein in a patient with 'Child's Class A' liver cirrhosis. J Minim Access Surg 2012;8:54-6.

9 van Buuren HR, Fick TE, Schalm SW. Recurrent bleeding from cutaneous venous collaterals in portal hypertension. Gut 1988;29:1279-81.

10 Singla V, Galwa RP, Saxena AK, et al. Cruveilhier Baumgarten syndrome with giant paraumbilical vein. J Postgrad Med 2008;54:328-9.

11 Palazon JM, Minguez M. Images in hepatology. Cruveilhier-Baumgarten syndrome. J Hepatol 1997;26:1413.

Copyright 2015 BMJ Publishing Group. All rights reserved. For permission to reuse any of this content visit

http://group.bmi.com/group/rights-licensing/permissions.

BMJ Case Report Fellows may re-use this article for personal use and teaching without any further permission.

Become a Fellow of BMJ Case Reports today and you can:

- Submit as many cases as you like

- Enjoy fast sympathetic peer review and rapid publication of accepted articles

- Access all the published articles

- Re-use any of the published material for personal use and teaching without further permission

For information on Institutional Fellowships contact consortiasales@bmjgroup.com

Visit casereports.bmj.com for more articles like this and to become a Fellow 